Theoretical Informatics and Applications

RAIRO: Theoret. Informatics Appl. 38 (2004) 49-67

DOI: $10.1051 /$ ita:2004003

\title{
FREE GROUP LANGUAGES: RATIONAL VERSUS RECOGNIZABLE*
}

\author{
PEDRo V. Silva ${ }^{1}$
}

\begin{abstract}
We provide alternative proofs and algorithms for results proved by Sénizergues on rational and recognizable free group languages. We consider two different approaches to the basic problem of deciding recognizability for rational free group languages following two fully independent paths: the symmetrification method (using techniques inspired by the study of inverse automata and inverse monoids) and the right stabilizer method (a general approach generalizable to other classes of groups). Several different algorithmic characterizations of recognizability are obtained, as well as other decidability results.
\end{abstract}

Mathematics Subject Classification. 20E05, 20F10, 68Q45.

\section{INTRODUCTION}

In [7] Sénizergues solved a conjecture raised by Sakarovitch in [5] and [6] by proving that every rational free group language is either recognizable or disjunctive (its syntactic congruence is the identity). In the process of doing so, Sénizergues showed also that recognizability is decidable for rational free group languages and their syntactic congruences are decidable.

Our purpose in the present paper is to provide further insight into rationality and recognizability of free group languages by considering two alternative approaches to the decidability of recognizability. These two approaches are independent from each other and from Sénizergues's strategy to solve the problem. In the end, we end up with alternative proofs for Sénizergues results obtained in a different order.

Keywords and phrases. Free group, rational subsets, recognizable subsets.

* Supported by F.C.T.

${ }^{1}$ Centro de Matemática, Faculdade de Ciências, Universidade do Porto, R. do Campo Alegre, 687, 4169-007 Porto, Portugal; e-mail: pvsilva@fc.up.pt 
We named the first of these approaches the symmetrification method. Symmetrification here means the automaton obtained from an automaton on a dual alphabet $\bar{X}=X \cup X^{-1}$ by adding dual edges to the original ones, a common technique in the context of inverse automata and inverse monoids [8]. We obtain here several different characterizations of recognizability, most of them algorithmic.

The second approach is fully independent of the first and is named the right stabilizer method. By right stabilizer of a language $L$ we mean the set of all elements $g$ such that $L g=L$. The largest normal subgroup contained in the right stabilizer is called syntactic subgroup in [7]. This approach may be generalized to other classes of groups ( $c f .[9])$.

Section 2 introduces notation and basic results of language theory. In Section 3 we introduce some basic results for group languages in general. Section 4 is devoted to the symmetrification method and Section 5 to the right stabilizer method.

\section{Preliminaries}

The reader is referred to [1] for definitions and results stated in this section. We remark that all the proofs presented in this paper are constructive in the sense that they provide algorithms to construct a rational expression, a finite automaton, etc. This fact will be always implicit when we shall refer to previous results.

Let $M$ be a monoid. We call a subset of $M$ an $M$-language. Whenever possible, brackets will be omitted in the representation of singleton sets. Given $A, B \subseteq M$, we write $A B=\{a b \mid a \in A, b \in B\}$ and we denote by $A^{*}$ the submonoid of $M$ generated by $A$.

We denote by Rat $M$ the smallest family $\mathcal{F}$ of $M$-languages such that:

- every finite $M$-language is in $\mathcal{F}$;

- if $A, B \in \mathcal{F}$, then $A \cup B, A B, A^{*} \in \mathcal{F}$.

The elements of Rat $M$ are called rational $M$-languages. Alternatively, an $M$ language $A$ is said to be rational if $A$ can be obtained from finite $M$-languages using the operators union, product and star a finite number of times.

Given $A \subseteq M$, we define a relation $\sim_{A}$ on $M$ by $x \sim_{A} y$ if

$$
\forall p, q \in M p x q \in A \Leftrightarrow p y q \in A .
$$

The relation $\sim_{A}$ is a congruence on $M$, the syntactic congruence of $A$. We say that $A$ is a recognizable $M$-language if the congruence $\sim_{A}$ has finite index. We denote the set of all recognizable $M$-languages by $\operatorname{Rec} M$. Alternatively, an $M$-language $A$ is recognizable if there exists some homomorphism $\varphi: M \rightarrow N$ into a finite monoid $N$ such that $A \varphi \varphi^{-1} \subseteq A$. In this case, we have necessarily $\operatorname{Ker} \varphi \subseteq \sim_{A}$, where $\operatorname{Ker} \varphi=\{(u, v) \in M \times M \mid u \varphi=v \varphi\}$. It is well known that $\operatorname{Rec} M$ constitutes a boolean algebra [1] (Prop. III.1.1). 
The following simple result will prove useful in forthcoming sections:

Lemma 2.1. Let $\varphi: M \rightarrow N$ be a surjective monoid homomorphism and let $A \subseteq N$. Write $\sigma=\sim_{A \varphi^{-1}}$ and $\tau=\sim_{A}$. Then

$$
M / \sigma \rightarrow N / \tau: x \sigma \mapsto(x \varphi) \tau
$$

is a monoid isomorphism.

Proof. Let $\Phi: M \rightarrow N / \tau$ be defined by $x \Phi=(x \varphi) \tau$. In view of the classical isomorphism theorems, it suffices to show that $\operatorname{Ker} \Phi=\sigma$. Given $x, y \in M$, we have

$$
\begin{aligned}
x \Phi=y \Phi & \Leftrightarrow(x \varphi) \tau(y \varphi) \\
& \Leftrightarrow \forall m, m^{\prime} \in M,(m \varphi)(x \varphi)\left(m^{\prime} \varphi\right) \in A \Leftrightarrow(m \varphi)(y \varphi)\left(m^{\prime} \varphi\right) \in A \\
& \Leftrightarrow \forall m, m^{\prime} \in M,\left(m x m^{\prime}\right) \varphi \in A \Leftrightarrow\left(m y m^{\prime}\right) \varphi \in A \\
& \Leftrightarrow \forall m, m^{\prime} \in M, m x m^{\prime} \in A \varphi^{-1} \Leftrightarrow m y m^{\prime} \in A \varphi^{-1} \\
& \Leftrightarrow x \sigma y
\end{aligned}
$$

and the lemma holds.

Classical language theory is restricted to the case where $M$ is a finitely generated free monoid $X^{*}$. In this context, recognizability can be most efficiently expressed through automata. Let $X$ be a finite set. Our notation for an $X$-automaton is a quadruple $\mathcal{A}=(Q, I, T, E)$ where $Q$ is a finite set, $I, T \subseteq Q$ and $E \subseteq Q \times X \times Q$. We may also write $Q=V(\mathcal{A})$ and $E=E(\mathcal{A})$ if convenient. The language $L(\mathcal{A}) \subseteq X^{*}$ recognized by $\mathcal{A}$ is the set of labels of successful paths in $\mathcal{A}$, that is, $u \in L(\mathcal{A})$ if and only if there exists a path in $\mathcal{A}$ of the form

$$
q_{0} \stackrel{x_{1}}{\longrightarrow} q_{1} \stackrel{x_{2}}{\longrightarrow} \ldots \stackrel{x_{n}}{\longrightarrow} q_{n}
$$

with $q_{0} \in I, q_{n} \in T,\left(q_{j-1}, x_{j}, q_{j}\right) \in E$ and $x_{1} \ldots x_{n}=u$. We shall use the notation $\rightarrow q$ (respectively $q \rightarrow$ ) to express that $q$ is an initial (respectively terminal) state. It is well known that $L \subseteq X^{*}$ is recognizable if and only if $L=L(\mathcal{A})$ for some $X$-automaton $\mathcal{A}$.

Kleene's Theorem states that $\operatorname{Rat} X^{*}=\operatorname{Rec} X^{*}$ for every finite set $X[1]$ (Prop. III.2.1). An easy consequence is that $\operatorname{Rec} M \subseteq$ Rat $M$ for every finitely generated monoid $M[1]$ (Prop. III.2.4).

Let $\mathcal{A}=(Q, I, T, E)$ be an $X$-automaton. We say that $\mathcal{A}$ is

- deterministic if $|I|=1$ and the implication

$$
(p, x, q),(p, x, r) \in E \Rightarrow q=r
$$

holds;

- trim if every vertex $q \in Q$ lies in some successful path;

- complete if the projection $E \rightarrow Q \times X:(p, x, q) \mapsto(p, x)$ is surjective.

If $\mathcal{A}$ is deterministic and $(p, x, q) \in E$, we write $q=p x$. 
It is well known that, given $L \in \operatorname{Rec} X^{*}$, there exists a unique, up to isomorphism, smallest deterministic $X$-automaton recognizing $L$, the minimum automaton of $L$. We denote the minimum automaton of $L$ by $\min _{L}$. If $L \neq \emptyset$, then $\min _{L}$ is trim. Moreover, $\min _{L}$ is a quotient of every trim deterministic $X$-automaton $\mathcal{A}$ recognizing $L$, that is, $\min _{L}$ can be obtained identifying vertices of $\mathcal{A}$ through an appropriate equivalence relation $\tau$. Indeed, if $\mathcal{A}=(Q, i, T, E), \tau$ is defined by

$$
p \tau q \Leftrightarrow L(Q, p, T, E)=L(Q, q, T, E) .
$$

Given $X$-automata $\mathcal{A}=(Q, I, T, E)$ and $\mathcal{A}^{\prime}=\left(Q^{\prime}, I^{\prime}, T^{\prime}, E^{\prime}\right)$, we define the direct product $\mathcal{A} \times \mathcal{A}^{\prime}=\left(Q \times Q^{\prime}, I \times I^{\prime}, T \times T^{\prime}, E^{\prime \prime}\right)$ by

$$
E^{\prime \prime}=\left\{\left(\left(p, p^{\prime}\right), x,\left(q, q^{\prime}\right)\right) \mid(p, x, q) \in E,\left(p^{\prime}, x, q^{\prime}\right) \in E^{\prime}\right\} .
$$

It is immediate that

$$
L\left(\mathcal{A} \times \mathcal{A}^{\prime}\right)=L(\mathcal{A}) \cap L\left(\mathcal{A}^{\prime}\right) .
$$

Moreover, if $\mathcal{A}$ and $\mathcal{A}^{\prime}$ are both deterministic, so is $\mathcal{A} \times \mathcal{A}^{\prime}$. We define also the trim subautomaton of $\mathcal{A}$ by

$$
\operatorname{tr}(\mathcal{A})=(Q \cap S, I \cap S, T \cap S, E \cap(S \times X \times S)),
$$

where $S$ consists of all vertices of $\mathcal{A}$ that lie in some successful path. Trivially, $L(\operatorname{tr}(\mathcal{A}))=L(\mathcal{A})$.

\section{Group languages}

For common concepts and results in group theory and combinatorial group theory, the reader is referred to [3] and [4], respectively. Most of the results included in this section are folklore, but we include some (simple) proofs.

Given a subgroup $H$ of a group $G$, we denote by $[G: H]$ the index of $H$ of $G$. We recall that a congruence $\sigma$ on a group $G$ is fully determined by the normal subgroup $1 \sigma$ : in fact,

$$
a \sigma b \Leftrightarrow a b^{-1} \in 1 \sigma
$$

holds for all $a, b \in G$ and $G / \sigma=G /(1 \sigma)$.

Given a group $G$ and some $A \subseteq G$, we define the right stabilizer of $A$ in $G$ to be

$$
R(A)=\{g \in G \mid A g \subseteq A\}
$$

We define also

$$
S(A)=\{g \in G \mid A g=A\}
$$

and

$$
N(A)=\bigcap_{x \in G} x(S(A)) x^{-1}
$$


Lemma 3.1. Let $G$ be a group and $A \subseteq G$. Then

(i) $S(A)=R(A) \cap(R(A))^{-1}$;

(ii) $S(A)$ is the largest subgroup of $G$ contained in $R(A)$;

(iii) $N(A)$ is the largest normal subgroup of $G$ contained in $S(A)$;

(iv) $N(A)=1 \sim_{A}$.

Proof. (i) Let $g \in S(A)$. Then $g \in R(A)$ trivially. Moreover, $A g^{-1}=A g g^{-1}=A$, hence $g^{-1} \in R(A)$ and so $g \in(R(A))^{-1}$. Thus $S(A) \subseteq R(A) \cap(R(A))^{-1}$.

Conversely, if $g \in R(A) \cap(R(A))^{-1}$, then $A g \subseteq \bar{A}$ and $A g^{-1} \subseteq A$. It follows that

$$
A g \subseteq A=A g^{-1} g \subseteq A g,
$$

thus $A g=A$ and $g \in S(A)$.

(ii) Clearly, $1 \in S(A)$ and

$$
g \in S(A) \Leftrightarrow A g=A \Leftrightarrow A=A g^{-1} \Leftrightarrow g^{-1} \in S(A)
$$

Let $g, h \in S(A)$. Then $A g h=A h=A$ and so $g h \in S(A)$. Thus $S(A)$ is a subgroup of $G$, and $S(A)$ is obviously contained in $R(A)$.

Let $H$ be another such subgroup. Given $h \in H$, we have then $h \in R(A)$. On the other hand, $h^{-1} \in H \subseteq R(A)$ yields $h \in(R(A))^{-1}$ and so $h \in R(A) \cap(R(A))^{-1}=$ $S(A)$. Therefore $H \subseteq S(A)$.

(iii) This is a standard group-theoretical construction.

(iv) Let $g \in N(A)$ and $x, y \in G$. We want to show that

$$
x g y \in A \Leftrightarrow x y \in A
$$

Suppose that $x g y \in A$. Since $g^{-1} \in N(A)$, we have $y^{-1} g^{-1} y \in N(A) \subseteq S(A)$, yielding

$$
x y=(x g y)\left(y^{-1} g^{-1} y\right) \in A \cdot S(A)=A .
$$

Conversely, $x y \in A$ yields

$$
x g y=(x y)\left(y^{-1} g y\right) \in A \cdot S(A)=A \text {. }
$$

Thus (2) holds and $N(A) \subseteq 1 \sim_{A}$.

To prove the converse inclusion, we only need to observe that $1 \sim_{A}$ is a normal subgroup of $G$ contained in $S(A)$ and apply (iii). Since $1 \sim_{A}$ is the kernel (in the group-theoretical sense) of a group homomorphism, it is certainly a normal subgroup of $G$. Finally, if $g \in 1 \sim_{A}$, then in particular

$$
a g \in A \Leftrightarrow a \in A
$$

It follows that $A g^{-1}=A$, hence $A g=A$ and $g \in S(A)$ as required.

The subgroup $N(A)$ is called in [7] the syntactic normal subgroup of $A$. 
Proposition 3.2. Let $G$ be a group and $A \subseteq G$. Then the following conditions are equivalent:
(i) $A \in \operatorname{Rec} G$;
(ii) $[G: N(A)]<\infty$;
(iii) $[G: S(A)]<\infty$.

Proof. We have $A \in \operatorname{Rec} G$ if and only if $\sim_{A}$ is a finite index congruence on $G$ if and only if $\left[G: 1 \sim_{A}\right]<\infty$. By Lemma 3.1(iv), we obtain (i) $\Leftrightarrow$ (ii). Since $N(A) \subseteq S(A)$, we have that (ii) $\Rightarrow$ (iii). It remains to prove that (iii) $\Rightarrow$ (ii).

Assume that $[G: S(A)]<\infty$. Then

$$
N(A)=\bigcap_{x \in G} x(S(A)) x^{-1}=\bigcap_{x \in G}(x S(A))\left(S(A) x^{-1}\right)
$$

is a finite intersection of finite index subgroups of $G$ and it follows easily that $[G: N(A)]<\infty$. Thus (ii) holds.

Since $S(H)=R(H)=H$, whenever $H$ is a subgroup of $G$, we obtain the following well-known result:

Corollary 3.3. If $H$ is a subgroup of $G$, then $H \in \operatorname{Rec} G$ if and only if $[G: H]<$ $\infty$.

Given a set $X$, let $X^{-1}=\left\{x^{-1} \mid x \in X\right\}$ be a set of formal inverses for $X$. We write $\bar{X}=X \cup X^{-1}$. We define an involution $\bar{X}^{*} \rightarrow \bar{X}^{*}: w \mapsto w^{-1}$ by:

$$
\begin{aligned}
& \left(x^{-1}\right)^{-1}=x \text { for every } x \in X \\
& \left(x_{1} \ldots x_{n}\right)^{-1}=x_{n}^{-1} \ldots x_{1}^{-1} \text { for all } x_{1}, \ldots x_{n} \in \bar{X}
\end{aligned}
$$

This involution is extended to $\bar{X}^{*}$-languages by defining

$$
L^{-1}=\left\{w^{-1} \mid w \in L\right\}
$$

It is an easy exercise to check that

$$
L \in \operatorname{Rat} \bar{X}^{*} \Rightarrow L^{-1} \in \operatorname{Rat} \bar{X}^{*} .
$$

This fact will be used without further reference.

A word $w \in \bar{X}^{*}$ is said to be reduced if $w$ contains no factor of the form $x x^{-1}$ for every $x \in \bar{X}$. We define a map $\iota: \bar{X}^{*} \rightarrow \bar{X}^{*}$ that assigns to every word $w \in \bar{X}^{*}$ the reduced word $w \iota$ obtained by successively cancelling from $w$ all factors of the form $x x^{-1}(x \in \bar{X})$. It is well known that

- $\iota$ is well defined;

- Ker $\iota$ is the congruence on $\bar{X}^{*}$ generated by the relation $\left\{\left(u u^{-1}, 1\right) \mid u \in\right.$ $\left.\bar{X}^{*}\right\}$;

- $F G(X)=\bar{X}^{*} /($ Ker $\iota)$ is the free group on $X$.

The canonical morphism $\bar{X}^{*} \rightarrow F G(X)$ will be denoted by $\pi$. 
We shall use the following version of Benois' Theorem:

Lemma 3.4 [1] (Prop. III.2.8). If $L \in \operatorname{Rat} \bar{X}^{*}$, then $L \iota \in \operatorname{Rat} \bar{X}^{*}$ and it is effectively constructible from $L$.

Finally, we relate rationality and recognizability in an arbitrary finitely generated group to the corresponding concepts in a free monoid $\bar{X}^{*}$.

Lemma 3.5. Let $G$ be a finitely generated group. Let $\alpha: F G(X) \rightarrow G$ be a surjective homomorphism for some finite set $X$ and write $\beta=\pi \alpha$. Then:

(i) $\operatorname{Rat} G=\left\{L \beta \mid L \in \operatorname{Rat} \bar{X}^{*}\right\}$;

(ii) given $L \in \operatorname{Rat} \bar{X}^{*}$, we have $L \beta \in \operatorname{Rec} G$ if and only if $L \beta \beta^{-1} \in \operatorname{Rat} \bar{X}^{*}$.

Proof. (i) Let $\mathcal{F}=\left\{L \beta \mid L \in \operatorname{Rat} \bar{X}^{*}\right\}$. Since a homomorphic image of a rational language is still rational [1] (Prop. III.2.2), we have $\mathcal{F} \subseteq$ Rat $G$. The converse inclusion follows from the straightforward fact that $\mathcal{F}$ contains the finite $G$-languages and is closed for the operators union, product and star.

(ii) Let $L \in \operatorname{Rat} \bar{X}^{*}$. By Lemma 2.1, we have

$$
G / \sim_{L \beta} \cong \bar{X}^{*} / \sim_{L \beta \beta^{-1}}
$$

Since $L \beta \in \operatorname{Rec} G$ if and only if $G / \sim_{L \beta}$ is finite and $L \beta \beta^{-1} \in \operatorname{Rat} \bar{X}^{*}$ if and only if $\bar{X}^{*} / \sim_{L \beta \beta^{-1}}$ is finite, the lemma follows.

\section{The SYMMETRIFICATION METHOD}

Let $\mathcal{A}=(Q, I, T, E)$ be an $\bar{X}$-automaton. We write

$$
D(E)=E \cup\left\{\left(q, x^{-1}, p\right) \mid(p, x, q) \in E\right\}
$$

and we define the $\bar{X}$-automaton $D(\mathcal{A})=(Q, I, T, D(E))$. We say that $D(\mathcal{A})$ is the symmetrification of $\mathcal{A}$. The automaton $\mathcal{A}$ is said to be inverse if

- $\mathcal{A}$ is deterministic;

- $\mathcal{A}$ is trim;

- $D(E)=E$.

An important example of inverse $\bar{X}$-automaton is the automaton $\mathcal{I}(H)=\left(Q_{H}, H, H, E_{H}\right)$ associated to a finite index subgroup $H$ of $F G(X)$ [2] (Sect. 2), where $Q_{H}$ is the set of all left cosets of $H$ and

$$
E_{H}=\{(H a, x, H a x) \mid a \in F G(X), x \in \bar{X}\} .
$$

In fact, $\mathcal{I}(H)$ is a complete inverse automaton and

$$
L(\mathcal{I}(H))=\left\{u \in \bar{X}^{*} \mid H(u \pi)=H\right\}=H \pi^{-1} .
$$

The following lemma will be useful. 
Lemma 4.1. Let $\mathcal{A}=(Q, i, T, E)$ be an inverse $\bar{X}$-automaton.

(i) If $p \stackrel{w}{\longrightarrow} q$ is a path in $\mathcal{A}$, then there exists also a path of the form $p \stackrel{w \iota}{\longrightarrow} q$.

(ii) If $(p, x, q),\left(p^{\prime}, x, q\right) \in E$, then $p=p^{\prime}$.

Proof. (i) Since $\mathcal{A}$ is inverse, all paths labelled by $y y^{-1}(y \in \bar{X})$ are loops. Since $w \iota$ is obtained from $w$ by successively removing factors of this form, the result follows.

(ii) If $(p, x, q),\left(p^{\prime}, x, q\right) \in E$, then we also have $\left(q, x^{-1}, p\right),\left(q, x^{-1}, p^{\prime}\right) \in E$ and the result follows from $\mathcal{A}$ being deterministic.

We are interested in obtaining different characterizations of recognizability for a rational $F G(X)$-language. In the next lemmas, we compare the alternative conditions we shall consider, starting with a useful technical statement.

Lemma 4.2. Let $L \in \operatorname{Rat} \bar{X}^{*}$ and let $\mathcal{A}=\min _{L \iota}$. If

$$
p \stackrel{u}{\longrightarrow} q, \quad p \stackrel{u}{\longrightarrow} r
$$

are paths in $\mathcal{A}$ and $D(\mathcal{A})$, respectively, then $q=r$.

Proof. Assume that

and

$$
p=q_{0} \stackrel{x_{1}}{\longrightarrow} q_{1} \stackrel{x_{2}}{\longrightarrow} \ldots \stackrel{x_{k}}{\longrightarrow} q_{k}=q
$$

$$
p=r_{0} \stackrel{x_{1}}{\longrightarrow} r_{1} \stackrel{x_{2}}{\longrightarrow} \ldots \stackrel{x_{k}}{\longrightarrow} r_{k}=r
$$

are paths in $\mathcal{A}$ and $D(\mathcal{A})$, respectively, with $u=x_{1} \ldots x_{k}$. We have $q_{0}=p=r_{0}$. Assume that $q_{j-1}=r_{j-1}$ for some $j \in\{1, \ldots, k\}$ and suppose that $q_{j} \neq r_{j}$. Then

$$
\left(q_{j-1}, x_{j}, q_{j}\right) \in E(\mathcal{A}), \quad\left(q_{j-1}, x_{j}, r_{j}\right) \in E(D(\mathcal{A})) .
$$

Since $\mathcal{A}$ is deterministic, we conclude that $\left(r_{j}, x_{j}^{-1}, q_{j-1}\right) \in E(\mathcal{A})$ and so we have a path in $\mathcal{A}$

$$
r_{j} \stackrel{x_{j}^{-1} x_{j}}{\longrightarrow} q_{j}
$$

labelled by a non reduced word, contradicting $\mathcal{A}=\min _{L \iota}$. Thus $r_{j}=q_{j}$. By induction, we conclude in particular that $r=r_{k}=q_{k}=q$.

We note that the preceding proof holds in fact for any finite deterministic trim automaton recognizing just reduced words.

Given a language $L$, we denote by $\operatorname{Pref}(L)$ the set of all prefixes of words in $L$. It is immediate that if $L$ is rational, so is $\operatorname{Pref}(L)$ - we only need to make all vertices terminal in $\min _{L}$.

Lemma 4.3. Let $L \in \operatorname{Rat} \bar{X}^{*}$ be nonempty and let $\mathcal{A}=\min _{L \iota}$. Consider the following conditions:
(A) $\operatorname{Pref}(L \iota)=\bar{X}^{*} \iota$;
(B) $\bar{X}^{*} / \sim_{L(D(\mathcal{A}))}$ is a group;
(C) $D(\mathcal{A})$ is complete. 
Then:

(i) $(A) \Rightarrow(C)$ and $(B) \Rightarrow(C)$;

(ii) no other implication holds in general.

Proof. (i) Assume that (A) holds. Let $p \in V(\mathcal{A})$ and $x \in \bar{X}$. Since $\mathcal{A}$ is trim, we have a path of the form $\rightarrow i \stackrel{u}{\longrightarrow} p$ in $\mathcal{A}$. If $x^{-1}$ is the last letter of $u$, then we have an edge of the form $\left(q, x^{-1}, p\right)$ in $\mathcal{A}$ and so $(p, x, q)$ is an edge in $D(\mathcal{A})$. Otherwise, $u x \in \bar{X}^{*} \iota=\operatorname{Pref}(L \iota)$ and so $u x v \in L \iota$ for some word $v$. Since $\mathcal{A}$ is deterministic, this implies the existence of an edge of the form $(p, x, r)$ in $\mathcal{A}$ (and consequently in $D(\mathcal{A})$ ). Thus $D(\mathcal{A})$ is complete and $(\mathrm{C})$ holds.

Assume now that $(\mathrm{B})$ holds. Let $p \in V(D(\mathcal{A}))=V(\mathcal{A})$ and $x \in \bar{X}$. Since $\mathcal{A}$ is trim, we have a path of the form

$$
\rightarrow i=p_{0} \stackrel{x_{1}}{\longrightarrow} p_{1} \stackrel{x_{2}}{\longrightarrow} \ldots \stackrel{x_{k}}{\longrightarrow} p_{k}=p
$$

in $\mathcal{A}$. Suppose first that $x_{k}=x^{-1}$. Then $\left(p, x, p_{k-1}\right) \in E(D(\mathcal{A}))$ as required. Thus we assume that $x^{-1} \neq x_{k}$. Since $\bar{X}^{*} / \sim_{L(D(\mathcal{A}))}$ is a finite group, there exists some $n>0$ such that $x^{n} \sim_{L(D(\mathcal{A}))}$ 1. Let $p \stackrel{v}{\longrightarrow} t \rightarrow$ be a path in $D(\mathcal{A})$ from $p$ onto a final vertex. Since $x_{1} \ldots x_{k} v \in L(D(\mathcal{A}))$ and $x^{n} \sim_{L(D(\mathcal{A}))} 1$, we obtain $x_{1} \ldots x_{k} x^{n} v \in L(D(\mathcal{A}))$. It follows that there exists a path of the form

$$
\rightarrow i=q_{0} \stackrel{x_{1}}{\longrightarrow} q_{1} \stackrel{x_{2}}{\longrightarrow} \ldots \stackrel{x_{k}}{\longrightarrow} q_{k} \stackrel{x}{\longrightarrow} q \stackrel{x^{n-1} v}{\longrightarrow} t^{\prime} \rightarrow
$$

in $D(\mathcal{A})$. Since $(3)$ is a path in $\mathcal{A}$, Lemma 4.2 yields $q_{k}=p_{k}=p$. Hence $(p, x, q) \in E(D(\mathcal{A}))$ and $D(\mathcal{A})$ is complete. Therefore $(\mathrm{C})$ holds.

(ii) Let $X=\{x\}$ and consider $L=x^{*}$. Then:

- $\operatorname{Pref}(L \iota)=x^{*}$ and so $(\mathrm{A})$ does not hold;

- $L(D(\mathcal{A}))=\bar{X}^{*}, \bar{X}^{*} / \sim_{L(D(\mathcal{A}))}$ is the trivial group and $D(\mathcal{A})$ is complete, thus (B) and (C) do both hold.

Therefore $(\mathrm{B}) \nRightarrow(\mathrm{A})$ and $(\mathrm{C}) \nRightarrow(\mathrm{A})$.

Finally, we consider $L^{\prime}=x^{+} \cup\left(x^{-1}\right)^{+}$. Then:

- $\operatorname{Pref}\left(L^{\prime} \iota\right)=\bar{X}^{*}$ and so $(\mathrm{A})$ holds;

- $L(D(\mathcal{A}))=\bar{x}^{+}$, hence $\bar{X}^{*} / \sim_{L(D(\mathcal{A}))}$ is a two-element semilattice and (B) does not hold;

- $D(\mathcal{A})$ is complete, thus $(\mathrm{C})$ holds.

Therefore $(\mathrm{A}) \nRightarrow(\mathrm{B})$ and $(\mathrm{C}) \nRightarrow(\mathrm{B})$.

Lemma 4.4. Let $L \in \operatorname{Rat} \bar{X}^{*}$ be nonempty and let $\mathcal{A}=\min _{L \iota}$. Suppose that $(L(D(\mathcal{A}))) \iota=L \iota$. Then conditions $(A),(B)$ and $(C)$ in the preceding lemma are equivalent.

Proof. $(\mathrm{A}) \Rightarrow(\mathrm{C})$. By Lemma 4.3(i).

$(\mathrm{C}) \Rightarrow(\mathrm{B})$. Assume that $(\mathrm{C})$ holds. Since the implication

$$
(p, x, q) \in E(D(\mathcal{A})) \Rightarrow\left(q, x^{-1}, p\right) \in E(D(\mathcal{A}))
$$


holds in $D(\mathcal{A})$, we conclude that the implication

$$
u v \in L(D(\mathcal{A})) \Rightarrow u x x^{-1} v \in L(D(\mathcal{A}))
$$

holds for all $u, v \in \bar{X}^{*}$ and $x \in \bar{X}$. Since $L \iota=L(\mathcal{A}) \subseteq L(D(\mathcal{A}))$ and every word in $L \pi \pi^{-1}$ can be obtained from a word in $L \iota$ by successively inserting factors of the form $x x^{-1}(x \in \bar{X})$, we obtain the inclusion $L \pi \pi^{-1} \subseteq L(D(\mathcal{A}))$.

Conversely,

$$
(L(D(\mathcal{A}))) \iota=L \iota \Rightarrow(L(D(\mathcal{A}))) \pi=L \pi \Rightarrow L(D(\mathcal{A})) \subseteq L \pi \pi^{-1},
$$

thus $L(D(\mathcal{A}))=L \pi \pi^{-1}$ and

$$
\bar{X}^{*} / \sim_{L(D(\mathcal{A}))}=\bar{X}^{*} / \sim_{L \pi \pi^{-1}} \cong F G(X) / \sim_{L \pi}
$$

by Lemma 2.1. Thus (B) holds.

$(\mathrm{B}) \Rightarrow(\mathrm{A})$. Assume that $(\mathrm{B})$ holds. Then we can fix some $w \in L \iota$. Let $u \in \bar{X}^{*} \iota-1$. Then $u$ is a prefix of a cyclically reduced word $v-$ a word whose square is reduced. Indeed, if $u$ is not itself cyclically reduced, then $u$ is of the form $x u^{\prime} x^{-1}$ for some $x \in \bar{X}$, implying that $|X|>1$. In this case, we can take $y \in X-x$ and consider $v=u y$. Since $\bar{X}^{*} / \sim_{L(D(\mathcal{A}))}$ is a (finite) group, there exists some $n>0$ such that $v^{n} \sim_{L(D(\mathcal{A}))} 1$. We may assume that $n>|w|$. Since $w \in L \iota \subseteq L(D(\mathcal{A}))$, we obtain $v^{n} w \in L(D(\mathcal{A}))$. Thus

$$
\left(v^{n} w\right) \iota \in(L(D(\mathcal{A}))) \iota=L \iota .
$$

Since $n>|w|$ and $v$ is cyclically reduced, we have $\left(v^{n} w\right) \iota=v z$ for some $z \in \bar{X}^{*} \iota$. Thus $u \in \operatorname{Pref}(v) \subseteq \operatorname{Pref}(L \iota)$ and so $\operatorname{Pref}(L \iota)=\bar{X}^{*} \iota$ as required.

Before proving the main theorem of the section, we need one more technical lemma.

Lemma 4.5. Let $L \in \operatorname{Rat} \bar{X}^{*}$ and let $\mathcal{A}=\min _{L \iota}$. If $D(\mathcal{A})$ is complete, then $L \pi \pi^{-1} \subseteq L(D(\mathcal{A}))$.

Proof. Since $L \iota=L(\mathcal{A}) \subseteq L(D(\mathcal{A}))$ and every word in $L \pi \pi^{-1}$ can be obtained from a word in $L \iota$ by successively inserting factors of the form $x x^{-1}(x \in \bar{X})$, it suffices to show that

$$
u v \in L(D(\mathcal{A})) \Rightarrow u x x^{-1} v \in L(D(\mathcal{A}))
$$

holds for all $u, v \in \bar{X}^{*}$ and $x \in \bar{X}$. Since the implication

$$
(p, x, q) \in E(D(\mathcal{A})) \Rightarrow\left(q, x^{-1}, p\right) \in E(D(\mathcal{A}))
$$

holds in $D(\mathcal{A})$, our claim follows from $D(\mathcal{A})$ being complete.

The following theorem provides four different characterizations of recognizability involving the symmetrification of $\min _{L \iota}$. 
Theorem 4.6. Let $L \in \operatorname{Rat} \bar{X}^{*}$ be nonempty and let $\mathcal{A}=\min _{L \iota}$. Then the following conditions are equivalent:

(i) $L \pi \in \operatorname{Rec} F G(X)$;

(ii) $(L(D(\mathcal{A}))) \iota=L \iota$ and $\operatorname{Pref}(L \iota)=\bar{X}^{*} \iota$;

(iii) $(L(D(\mathcal{A}))) \iota=L \iota$ and $\bar{X}^{*} / \sim_{L(D(\mathcal{A}))}$ is a group;

(iv) $(L(D(\mathcal{A}))) \iota=L \iota$ and $D(\mathcal{A})$ is complete;

(v) $\operatorname{Pref}(L \iota)=\bar{X}^{*} \iota$ and $\bar{X}^{*} / \sim_{L(D(\mathcal{A}))}$ is a group.

Proof. By Lemma 4.4, conditions (ii), (iii) and (iv) are equivalent.

We prove next that (i) implies (iv). Assume that $L \pi \in \operatorname{Rec} F G(X)$ and write $H=1 \sim_{L \pi}$. Since $F G(X) / H=F G(X) / \sim_{L \pi}, H$ is a finite index subgroup of $F G(X)$. We observed before that $\mathcal{I}(H)=\left(Q_{H}, H, H, E_{H}\right)$ is a complete inverse automaton. Let $\mathcal{B}=\left(Q_{H}, H, T, E_{H}\right)$, with

$$
T=\{H a \mid a \in L \pi\} .
$$

Since $L \neq \emptyset$, then $T \neq \emptyset$. Hence $\mathcal{B}$ is trim and so is a complete inverse automaton like $\mathcal{I}(H)$. It is immediate that

$$
L(\mathcal{B})=\left\{u \in \bar{X}^{*} \mid H(u \pi) \in T\right\}=\left\{u \in \bar{X}^{*} \mid H(u \pi)=H(v \pi) \text { for some } v \in L\right\} .
$$

Now

$$
H(u \pi)=H(v \pi) \Leftrightarrow\left(u v^{-1}\right) \pi \in H \Leftrightarrow\left(u v^{-1}\right) \pi \sim_{L \pi} 1 \Leftrightarrow u \pi \sim_{L \pi} v \pi .
$$

Since $u \pi \sim_{L \pi} v \pi$ for some $v \in L$ if and only if $u \pi \in L \pi$, we conclude that $L(\mathcal{B})=L \pi \pi^{-1}$. Since $\mathcal{B}$ is deterministic and trim, we know that $\mathcal{B}^{\prime}=\min _{L \pi \pi^{-1}}$ must be a quotient of $\mathcal{B}$ (that is, obtained from $\mathcal{B}$ by identification of vertices). It is not difficult to check that all the properties characterizing a complete inverse automaton are preserved by quotients, with the possible exception of determinism. Since $\mathcal{B}^{\prime}$ is deterministic by definition, then $\mathcal{B}^{\prime}$ is itself complete and inverse. We write $\mathcal{B}^{\prime}=\left(Q^{\prime}, i^{\prime}, T^{\prime}, E^{\prime}\right)$.

We define now the $\bar{X}$-automaton $\mathcal{R}=\left(\bar{X} \cup 1,1, \bar{X} \cup 1, E_{R}\right)$, where

$$
E_{R}=\left\{(y, x, x) \in(\bar{X} \cup 1) \times \bar{X} \times \bar{X} \mid y \neq x^{-1}\right\} .
$$

It is easy to check that $\mathcal{R}=\min _{\bar{X}}{ }^{*} \iota$. We show that

$$
\mathcal{A} \cong \operatorname{tr}\left(\mathcal{B}^{\prime} \times \mathcal{R}\right) \text {. }
$$

Let $\mathcal{C}=\operatorname{tr}\left(\mathcal{B}^{\prime} \times \mathcal{R}\right)$. Since $\mathcal{B}^{\prime}$ and $\mathcal{R}$ are both deterministic, so is $\mathcal{C}$. Moreover,

$$
L(\mathcal{C})=L\left(\mathcal{B}^{\prime} \times \mathcal{R}\right)=L\left(\mathcal{B}^{\prime}\right) \cap L(\mathcal{R})=L \pi \pi^{-1} \cap \bar{X}^{*} \iota=L \iota,
$$

thus we conclude that $\mathcal{C}$ is a trim deterministic automaton recognizing $L \iota$. To show that $\mathcal{C}=\min _{L \iota}=\mathcal{A}$, it remains to show that the equivalence $\tau$ defined in 
the vertex set of $\mathcal{C}$ by (1) is the identity. Write $\mathcal{C}=\left(Q_{C}, i_{C}, T_{C}, E_{C}\right)$ and let $p, q \in Q_{C}$ be distinct. We must show that $L\left(Q_{C}, p, T_{C}, E_{C}\right) \neq L\left(Q_{C}, q, T_{C}, E_{C}\right)$. Since $\mathcal{C}$ is a subautomaton of $\mathcal{B}^{\prime} \times \mathcal{R}$, we may write $p=\left(p_{1}, p_{2}\right)$ and $q=\left(q_{1}, q_{2}\right)$ with $p_{1}, q_{1} \in V\left(\mathcal{B}^{\prime}\right)=Q^{\prime}$ and $p_{2}, q_{2} \in V(\mathcal{R})=\bar{X} \cup 1$.

Consider first the case $p_{2} \neq q_{2}$. Interchanging $p$ and $q$ if necessary, we may assume that there exists some $x \in \bar{X}$ such that $p_{2} x$ is defined in $\mathcal{R}$ but $q_{2} x$ is not - equivalently, $p_{2} x$ is a reduced word but $q_{2} x$ is not.

We remark now that we can always reach a terminal vertex from any vertex of $\mathcal{B}^{\prime} \times \mathcal{R}$. In fact, let $\left(s_{1}, s_{2}\right) \in V\left(\mathcal{B}^{\prime} \times \mathcal{R}\right)$. Take $m=\left|Q^{\prime}\right|$ and take $y \in \bar{X}-s_{2}^{-1}$. Since $\mathcal{B}^{\prime}$ is complete, we have a path

$$
s_{1} \stackrel{y^{m}}{\longrightarrow} r_{1}
$$

in $\mathcal{B}^{\prime}$ for some vertex $r_{1}$. Since $\mathcal{B}^{\prime}$ is trim, we have a path

$$
r_{1} \stackrel{u}{\longrightarrow} t_{1} \rightarrow
$$

in $\mathcal{B}^{\prime}$ onto some terminal vertex $t_{1}$. We may assume of course that $|u|<\left|Q^{\prime}\right|$. We conclude that there exists a path

$$
s_{1} \stackrel{y^{m} u}{\longrightarrow} t_{1} \rightarrow
$$

in $\mathcal{B}^{\prime}$. By Lemma 4.1(i), there exists a path of the form

$$
s_{1} \stackrel{\left(y^{m} u\right) \iota}{\longrightarrow} t_{1} \rightarrow
$$

Since $m>|u|$, we have $\left(y^{m} u\right) \iota=y v$ for some reduced word $v$. Since $y \neq s_{2}^{-1}$, we must have a path

$$
\left(s_{1}, s_{2}\right) \stackrel{y v}{\longrightarrow}\left(t_{1}, t_{2}\right) \rightarrow
$$

in $\mathcal{B}^{\prime} \times \mathcal{R}$ for some $t_{2} \in V(\mathcal{R})$.

Now, since $\mathcal{B}^{\prime}$ is complete, $p_{1} x$ is defined and the preceding remark implies that we have a path

$$
\left(p_{1}, p_{2}\right) \stackrel{x w}{\longrightarrow}\left(t_{1}, t_{2}\right) \rightarrow
$$

in $\mathcal{B}^{\prime} \times \mathcal{R}$ (and therefore in $\mathcal{C}$ ) for some terminal vertex $\left(t_{1}, t_{2}\right)$. However, since $q_{2} x$ is not defined in $\mathcal{R}$, we have no path of the form

$$
\left(q_{1}, q_{2}\right) \stackrel{x w}{\longrightarrow}\left(t_{1}^{\prime}, t_{2}^{\prime}\right) \rightarrow
$$

in $\mathcal{C}$. Thus $L\left(Q_{C}, p, T_{C}, E_{C}\right) \neq L\left(Q_{C}, q, T_{C}, E_{C}\right)$ in this case.

Assume now that $p_{2}=q_{2}$. Thus $p_{1} \neq q_{1}$. Since $\mathcal{B}^{\prime}$ is a minimal automaton, the languages $L\left(Q^{\prime}, r^{\prime}, T^{\prime}, E^{\prime}\right)\left(r^{\prime} \in Q^{\prime}\right)$ are all different. Thus there exists some $M \in \mathbb{N}$ such that the words in

$$
\left\{w \in \bar{X}^{*}|| w \mid<M\right\}
$$


suffice to distinguish all of them. Take $x \in \bar{X}$ such that $p_{2} x$ is defined in $\mathcal{R}$. Since $\mathcal{B}^{\prime}$ is complete, $p_{1} x^{M}$ and $q_{1} x^{M}$ are both defined. By Lemma 4.1(ii), $p_{1} \neq q_{1}$ implies $p_{1} x^{M} \neq q_{1} x^{M}$. Without loss of generality, we may then assume that there exists some $w \in L\left(Q^{\prime}, p_{1} x^{M}, T^{\prime}, E^{\prime}\right)-L\left(Q^{\prime}, q_{1} x^{M}, T^{\prime}, E^{\prime}\right)$ with $|w|<M$. Since $\mathcal{B}^{\prime}$ is complete, we have in fact $w \in L\left(Q^{\prime}, q_{1} x^{M}, Q^{\prime}-T^{\prime}, E^{\prime}\right)$. Thus

$$
x^{M} w \in L\left(Q^{\prime}, p_{1}, T^{\prime}, E^{\prime}\right), \quad x^{M} w \in L\left(Q^{\prime}, q_{1}, Q^{\prime}-T^{\prime}, E^{\prime}\right)
$$

and Lemma 4.1(i) yields

$$
\left(x^{M} w\right) \iota \in L\left(Q^{\prime}, p_{1}, T^{\prime}, E^{\prime}\right), \quad\left(x^{M} w\right) \iota \in L\left(Q^{\prime}, q_{1}, Q^{\prime}-T^{\prime}, E^{\prime}\right) .
$$

Since $|w|<M$, we have $\left(x^{M} w\right) \iota=x u$ for some reduced word $u$.

Since $p_{2} x$ is defined in $\mathcal{R}$, we have a path

$$
\left(p_{1}, p_{2}\right) \stackrel{x u}{\longrightarrow}\left(t_{1}, t_{2}\right) \rightarrow
$$

in $\mathcal{C}$ for some $t_{1} \in T^{\prime}$ and $t_{2} \in V(\mathcal{R})$. However, since $q_{1} x u \notin T^{\prime}$, we have no path of the form

$$
\left(q_{1}, q_{2}\right) \stackrel{x v}{\longrightarrow}\left(t_{1}^{\prime}, t_{2}^{\prime}\right) \rightarrow
$$

in $\mathcal{C}$. Thus $L\left(Q_{C}, p, T_{C}, E_{C}\right) \neq L\left(Q_{C}, q, T_{C}, E_{C}\right)$ also in this case and (4) holds.

We show now that $D(\mathcal{A})$ is complete. By (4), we may assume that $\mathcal{A}=\operatorname{tr}\left(\mathcal{B}^{\prime} \times\right.$ $\mathcal{R})$. Let $\left(p_{1}, p_{2}\right) \in V(D(\mathcal{A}))=V(\mathcal{A})$ and $x \in \bar{X}$. Since $\mathcal{A}$ is trim, we have a path of the form

$$
\rightarrow\left(i^{\prime}, 1\right) \stackrel{w}{\longrightarrow}\left(p_{1}, p_{2}\right)
$$

in $\mathcal{A}$. Suppose first that the last letter of $w$ is $x^{-1}$. Then we have an edge $\left(\left(q_{1}, q_{2}\right), x^{-1},\left(p_{1}, p_{2}\right)\right)$ in $\mathcal{A}$ and so $\left(\left(p_{1}, p_{2}\right), x,\left(q_{1}, q_{2}\right)\right) \in E(D(\mathcal{A}))$ as required. Suppose now that $x^{-1}$ is not the last letter of $w$. Then we have an edge $\left(p_{2}, x, x\right)$ in $\mathcal{R}$. On the other hand, since $\mathcal{B}^{\prime}$ is complete, we have an edge of the form $\left(p_{1}, x, q_{1}\right)$ in $\mathcal{B}^{\prime}$. Thus $\left(\left(p_{1}, p_{2}\right), x,\left(q_{1}, x\right)\right) \in E\left(\mathcal{B}^{\prime} \times \mathcal{R}\right)$. We remarked before that we can always reach a terminal vertex from an arbitrary vertex of $\mathcal{B}^{\prime} \times \mathcal{R}$, hence $\left(q_{1}, x\right) \in V(\mathcal{C})$ and so $\left(\left(p_{1}, p_{2}\right), x,\left(q_{1}, x\right)\right) \in E(\mathcal{A}) \subseteq E(D(\mathcal{A}))$. Thus $D(\mathcal{A})$ is complete.

Next we show that

$$
L(D(\mathcal{A}))=L \pi \pi^{-1}
$$

Let $u \in L(D(\mathcal{A}))$. Then we have a successful path

$$
\rightarrow\left(p_{0}, q_{0}\right) \stackrel{x_{1}}{\longrightarrow}\left(p_{1}, q_{1}\right) \stackrel{x_{2}}{\longrightarrow} \ldots \stackrel{x_{n}}{\longrightarrow}\left(p_{n}, q_{n}\right) \rightarrow
$$

in $D(\mathcal{A})$ with $x_{1} \ldots x_{n}=u$. Let $j \in\{1, \ldots, n\}$. By definition of $D(\mathcal{A})$, we have either

$$
\left(\left(p_{j-1}, q_{j-1}\right), x_{j},\left(p_{j}, q_{j}\right)\right) \in E(\mathcal{A}) \quad \text { or } \quad\left(\left(p_{j}, q_{j}\right), x_{j}^{-1},\left(p_{j-1}, q_{j-1}\right)\right) \in E(\mathcal{A}) \text {. }
$$


Hence $\left(p_{j-1}, x_{j}, p_{j}\right) \in E\left(\mathcal{B}^{\prime}\right)$ or $\left(p_{j}, x_{j}^{-1}, p_{j-1}\right) \in E\left(\mathcal{B}^{\prime}\right)$. Since $\mathcal{B}^{\prime}$ is inverse, we get $\left(p_{j-1}, x_{j}, p_{j}\right) \in E\left(\mathcal{B}^{\prime}\right)$ in either case and so we have a path

$$
\rightarrow p_{0} \stackrel{x_{1}}{\longrightarrow} p_{1} \stackrel{x_{2}}{\longrightarrow} \ldots \stackrel{x_{n}}{\longrightarrow} p_{n} \rightarrow
$$

in $\mathcal{B}^{\prime}$, showing that $u \in L\left(\mathcal{B}^{\prime}\right)=L \pi \pi^{-1}$. Thus $L(D(\mathcal{A})) \subseteq L \pi \pi^{-1}$.

The reverse inclusion follows from Lemma 4.5 , hence $(5)$ holds. Thus

$$
(L(D(\mathcal{A}))) \iota=\left(L \pi \pi^{-1}\right) \iota=L \iota
$$

and so condition (iv) holds.

Conversely, assume that condition (iv) holds. We show that (i) holds. By Lemma 3.5(ii), we must show that $L \pi \pi^{-1} \in \operatorname{Rat} \bar{X}^{*}$. Therefore it suffices to show that $L \pi \pi^{-1}=L(D(\mathcal{A}))$.

Since $\operatorname{Ker} \iota=\operatorname{Ker} \pi$, we have $(L(D(\mathcal{A}))) \pi=L \pi$ and so $L(D(\mathcal{A})) \subseteq L \pi \pi^{-1}$. The converse inclusion follows from Lemma 4.5. Therefore (i) holds and so (i) is equivalent to (iv).

Since (ii) is equivalent to (iii), it is clear that $(\mathrm{v})$ is implied by the other conditions. It remains to prove that $(\mathrm{v})$ implies $(L(D(\mathcal{A}))) \iota=L \iota$. We assume then that (v) holds. Since $L \iota \subseteq(L(D(\mathcal{A}))) \iota$ trivially, we only have to prove the opposite inclusion. We start showing that

$$
u x x^{-1} v \in L(D(\mathcal{A})) \Rightarrow u v \in L(D(\mathcal{A}))
$$

holds for all $u, v \in \bar{X}^{*}$ and $x \in \bar{X}$. Since $\bar{X}^{*} / \sim_{L(D(\mathcal{A}))}$ is a (finite) group, there exists some $n>0$ such that

$$
x^{n} \sim_{L(D(\mathcal{A}))} 1 \sim_{L(D(\mathcal{A}))} x^{-n} .
$$

On the other hand, by Lemma $4.3(\mathrm{i})$, we know that $D(\mathcal{A})$ is complete and so we can always insert a loop labelled by $x^{n-1} x^{1-n}$ at any position in an arbitrary path in $D(\mathcal{A})$. Thus

$u x x^{-1} v \in L(D(\mathcal{A})) \Rightarrow u x^{n} x^{-n} v \in L(D(\mathcal{A})) \Rightarrow u x^{-n} v \in L(D(\mathcal{A})) \Rightarrow u v \in L(D(\mathcal{A}))$ and (6) holds.

Let $w \in L(D(\mathcal{A}))$. By $(6)$, we have $w \iota \in L(D(\mathcal{A}))$. Write $w \iota=x_{1} \ldots x_{k}$, with $x_{1}, \ldots, x_{k} \in \bar{X}$. Since $L \neq \emptyset$, then $\operatorname{Pref}(L \iota)=\bar{X}^{*} \iota$. Thus there exists some $z \in \bar{X}^{*} \iota$ such that $(w \iota) z \in L \iota$. Therefore we have paths of the form

$$
\begin{gathered}
\rightarrow i=p_{0} \stackrel{x_{1}}{\longrightarrow} p_{1} \stackrel{x_{2}}{\longrightarrow} \ldots \stackrel{x_{k}}{\longrightarrow} p_{k} \rightarrow, \\
\rightarrow i=q_{0} \stackrel{x_{1}}{\longrightarrow} q_{1} \stackrel{x_{2}}{\longrightarrow} \ldots \stackrel{x_{k}}{\longrightarrow} q_{k} \stackrel{z}{\longrightarrow} t \rightarrow
\end{gathered}
$$

in $D(\mathcal{A})$ and $\mathcal{A}$, respectively. By Lemma 4.2 , we obtain $q_{k}=p_{k}$, hence $w \iota \in L \iota$ and $(L(D(\mathcal{A}))) \iota \subseteq L \iota$ as required. Therefore $(L(D(\mathcal{A}))) \iota=L \iota$ and the theorem is proved. 
Together with Lemma 4.3(ii), the next example shows that we cannot omit any of the conditions appearing in (ii)-(v) of the preceding theorem.

Example 4.7. Let $X=\{x\}$ and $L=x$. Then $(L(D(\mathcal{A}))) \iota=L \iota$ but $\operatorname{Pref}(L \iota) \neq$ $\bar{X}^{*} \iota$.

Proof. Straightforward computation shows that $L(D(\mathcal{A}))=\left(x x^{-1}\right)^{*} x$. Thus $(L(D(\mathcal{A}))) \iota=x=L \iota$. However,

$$
\operatorname{Pref}(L \iota)=\{1, x\} \neq \bar{X}^{*} \iota .
$$

We can now deduce the following result, proved in the end of Section 3 of [7].

Corollary 4.8 [7]. Given $L \in \operatorname{Rat} \bar{X}^{*}$, it is decidable whether or not $L \pi \in$ $\operatorname{Rec} F G(X)$.

Proof. If $L=\emptyset$, then $L \pi=\emptyset \in \operatorname{Rec} F G(X)$. Assume that $L \neq \emptyset$. In view of Lemma 3.4, the automaton $\mathcal{A}$ can be effectively constructed, and so can $D(\mathcal{A})$. Therefore any one of the conditions (ii)-(v) in Theorem 4.6 can be checked using the basic decidability properties of rational languages.

The following corollary provides yet another characterization of recognizability involving $D(\mathcal{A})$, less interesting because nonalgorithmic.

Corollary 4.9. Let $L \in R a t \bar{X}^{*}$ and let $\mathcal{A}=\min _{L \iota}$. Then the following conditions are equivalent:

(i) $L \pi \in \operatorname{Rec} F G(X)$;

(ii) $L \pi \pi^{-1} \in \operatorname{Rat} \bar{X}^{*}$;

(iii) $L \pi \pi^{-1}=L(D(\mathcal{A}))$.

Proof. The equivalence (i) $\Leftrightarrow$ (ii) follows from Lemma 3.5(ii). The implication (i) $\Rightarrow$ (iii) follows from (5) in the proof of Theorem 4.6 for $L \neq \emptyset$. In the case $L=\emptyset$, it is immediate. Finally, we have (iii) $\Rightarrow$ (ii) trivially.

We can also obtain the following result, also proved in the end of Section 3 of $[7]$.

Corollary 4.10. Let $L \in R a t \bar{X}^{*}$ be such that $L \pi \in \operatorname{Rec} F G(X)$. Then the syntactic congruence $\sim_{L \pi}$ is decidable and the syntactic group $F G(X) / \sim_{L \pi}$ is effectively constructible.

Proof. By Lemma 2.1, we have a monoid isomorphism

$$
\bar{X}^{*} / \sim_{L \pi \pi^{-1}} \rightarrow F G(X) / \sim_{L \pi}: w \sim_{L \pi \pi^{-1}} \mapsto(w \pi) \sim_{L \pi} .
$$

By Corollary 4.9, we have $L \pi \pi^{-1}=L(D(\mathcal{A}))$. Thus, to decide whether or not $(u \pi) \sim_{L \pi}(v \pi)$ for some given $u, v \in \bar{X}^{*}$, we only need to check if $u \sim_{L(D(\mathcal{A}))} v$, and this can certainly be done because $L(D(\mathcal{A}))$ is an effectively constructible rational language. Therefore $\sim_{L \pi}$ is decidable. 
Since

$$
F G(X) / \sim_{L \pi} \cong \bar{X}^{*} / \sim_{L(D(\mathcal{A}))}
$$

we conclude that $F G(X) / \sim_{L \pi}$ is in fact effectively constructible.

\section{THE RIGHT STABILIZER METHOD}

An alternative (and shorter) path to the recursiveness of $\operatorname{Rec} F G(X)$ is given by the computation of right stabilizers. The following result is the cornerstone of the whole approach.

Theorem 5.1. Let $L, L^{\prime} \in \operatorname{Rat} \bar{X}^{*}$ and define

$$
P=\left\{u \in \bar{X}^{*} \iota \mid(L u) \iota \subseteq L^{\prime} \iota\right\}
$$

Then $P \in \operatorname{Rat} \bar{X}^{*}$.

Proof. Given $u \in \bar{X}^{*} \iota$, we have that

$$
\begin{aligned}
u \in P & \Leftrightarrow(L u) \iota \cap\left(\bar{X}^{*} \iota-L^{\prime} \iota\right)=\emptyset \\
& \Leftrightarrow \neg\left((L u) \iota \cap\left(\bar{X}^{*} \iota-L^{\prime} \iota\right) \neq \emptyset\right) \\
& \Leftrightarrow \neg\left(u \in\left(L^{-1}\left(\bar{X}^{*} \iota-L^{\prime} \iota\right)\right) \iota\right) \\
& \Leftrightarrow u \in \bar{X}^{*} \iota-\left(L^{-1}\left(\bar{X}^{*} \iota-L^{\prime} \iota\right)\right) \iota,
\end{aligned}
$$

thus $P=\bar{X}^{*} \iota-\left(L^{-1}\left(\bar{X}^{*} \iota-L^{\prime} \iota\right)\right) \iota$ is an effectively constructible rational $\bar{X}$ language by Lemma 3.4 and the standard closure properties of rational languages.

Now we are able to prove:

Theorem 5.2. Let $L \in \operatorname{Rat} \bar{X}^{*}$. Then $(R(L \pi)) \pi^{-1} \iota,(S(L \pi)) \pi^{-1} \iota \in \operatorname{Rat} \bar{X}^{*}$ and are effectively constructible from $L$.

Proof. Since $R(\emptyset)=S(\emptyset)=F G(X)$, we may assume that $L \neq \emptyset$. In view of Lemma 3.4 and $L \iota \pi=L \pi$, we may also assume that $L \subseteq \bar{X}^{*} \iota$. Write

$$
R^{\prime}=(R(L \pi)) \pi^{-1} \iota
$$

We have

$$
\begin{aligned}
R^{\prime} & =(R(L \pi)) \pi^{-1} \cap \bar{X}^{*} \iota=\left\{w \in \bar{X}^{*} \iota \mid(L \pi)(w \pi) \subseteq L \pi\right\} \\
& =\left\{w \in \bar{X}^{*} \iota \mid(L w) \iota \subseteq L\right\}
\end{aligned}
$$

hence $R^{\prime}$ is an effectively constructible rational language by Theorem 5.1.

Write

$$
S^{\prime}=(S(L \pi)) \pi^{-1} \iota \text {. }
$$


We have

$$
\begin{aligned}
S^{\prime} & =(S(L \pi)) \pi^{-1} \cap \bar{X}^{*} \iota=\left\{w \in \bar{X}^{*} \iota \mid(L \pi)(w \pi)=L \pi\right\} \\
& =\left\{w \in \bar{X}^{*} \iota \mid(L w) \iota=L\right\}
\end{aligned}
$$

Since

$$
(L w) \iota=L \Leftrightarrow(L w) \iota \subseteq L \wedge L \subseteq(L w) \iota \Leftrightarrow(L w) \iota \subseteq L \wedge\left(L w^{-1}\right) \iota \subseteq L,
$$

we conclude that

$$
S^{\prime}=R^{\prime} \cap\left(R^{\prime}\right)^{-1}
$$

Therefore $S^{\prime}$ is also an effectively constructible rational language.

The following lemma prepares us for a new characterization for recognizability.

Lemma 5.3. Let $H$ be a subgroup of $F G(X)$. Then $[F G(X): H]<\infty$ if and only if $H \pi^{-1} \iota \in \operatorname{Rat} \bar{X}^{*}$ and $\operatorname{Pref}\left(H \pi^{-1} \iota\right)=\bar{X}^{*} \iota$.

Proof. Suppose first that $[F G(X): H]<\infty$. We observed before that $\mathcal{I}(H)$ is a complete inverse automaton recognizing $H \pi^{-1}$. By Lemma 3.4, we conclude that $H \pi^{-1} \iota \in \operatorname{Rat} \bar{X}^{*}$. Let $u \in \bar{X}^{*} \iota$. If $u$ is not itself cyclically reduced, then $|X|>1$. In any case, there is some $x \in X \cup 1$ such that $u x$ is a nonempty cyclically reduced word. Let $n=[F G(X): H]=|V(\mathcal{I}(H))|$. Since $\mathcal{I}(H)$ is complete and trim, it contains a path of the form

$$
\rightarrow i \stackrel{(u x)^{n}}{\longrightarrow} q \stackrel{v}{\longrightarrow} i \rightarrow
$$

We can assume that $|v|<n$. By Lemma 4.1(i), we obtain

$$
\left((u x)^{n} v\right) \iota \in L(\mathcal{I}(H))=H \pi^{-1} .
$$

Since $u x \neq 1$ and $|v|<n$, we have $\left((u x)^{n} v\right) \iota=u w$ for some $w \in \bar{X}^{*} \iota$. Thus $u w=(u w) \iota \in H \pi^{-1} \iota$ and $u \in \operatorname{Pref}\left(H \pi^{-1} \iota\right)$.

Suppose now that $H \pi^{-1} \iota \in \operatorname{Rat} \bar{X}^{*}$ and $\operatorname{Pref}\left(H \pi^{-1} \iota\right)=\bar{X}^{*} \iota$. Let $\mathcal{A}=$ $(Q, i, T, E)$ denote $\min _{H \pi^{-1} \iota}$. For every $q \in Q$, we fix a path of the form

$$
q \stackrel{w_{q}}{\longrightarrow} t \in T \text {. }
$$

We show that

$$
F G(X)=\bigcup_{q \in Q} H\left(w_{q}^{-1} \pi\right)
$$

Let $u \in \bar{X}^{*} \iota$. Since $\operatorname{Pref}\left(H \pi^{-1} \iota\right)=\bar{X}^{*} \iota$, there exists a path in $\mathcal{A}$ of the form $i \stackrel{u}{\longrightarrow} q$. It follows that $u w_{q} \in L(\mathcal{A})=H \pi^{-1} \iota$ and so $\left(u w_{q}\right) \pi \in H \pi^{-1} \iota \pi=H$. Thus

$$
u \pi=\left(u w_{q} w_{q}^{-1}\right) \pi \in H\left(w_{q}^{-1} \pi\right)
$$

and (7) holds. Therefore $[F G(X): H]<\infty$ as required. 
Corollary 5.4. Given $L \in \operatorname{Rat} \bar{X}^{*}$, the following conditions are equivalent:

(i) $L \pi \in \operatorname{Rec} F G(X)$;

(ii) $[F G(X): S(L \pi)]<\infty$;

(iii) $\operatorname{Pref}\left((S(L \pi)) \pi^{-1} \iota\right)=\bar{X}^{*} \iota$.

Proof. The equivalence (i) $\Leftrightarrow$ (ii) follows from Proposition 3.2 and the implication (ii) $\Rightarrow$ (iii) follows from Lemma 5.3. Finally, assume that (iii) holds. Since $(S(L \pi)) \pi^{-1} \iota \in \operatorname{Rat} \bar{X}^{*}$ by Theorem 5.2 , we obtain $[F G(X): S(L \pi)]<\infty$ by Lemma 5.3. Thus (iii) $\Rightarrow$ (ii) as required.

An alternative algorithm for Corollary 4.8 follows, since condition (iii) in Corollary 5.4 is decidable in view of Theorem 5.2.

We give now an alternative proof for Sénizergues's theorem on rational $F G(X)$ languages, that proved in its full generality the conjecture raised by Sakarovitch in [5] and [6].

Corollary 5.5 [7] (Th. 1.1). Given $A \in \operatorname{Rat} F G(X)$, then either $A \in \operatorname{Rec} F G(X)$ or $\sim_{A}=i d$.

Proof. Let $A \in \operatorname{Rat} F G(X)$. By Lemma 3.5(i), we have $A=L \pi$ for some $L \in$ Rat $\bar{X}^{*}$. Assume that $A \notin \operatorname{Rec} F G(X)$. By Lemma 3.1(iv), we must show that $N(A)=1$. By Corollary 5.4, we must have $\operatorname{Pref}\left((S(A)) \pi^{-1} \iota\right) \neq \bar{X}^{*} \iota$. Take $u \in \bar{X}^{*} \iota-\operatorname{Pref}\left((S(A)) \pi^{-1} \iota\right)$ and let $v \in \bar{X}^{*} \iota-1$. We need to show that $v \pi \notin N(A)$.

Consider first the case $|X|=1$. Since $v$ is reduced and nonempty, there exists some nonzero integer $n$ such that $u$ is a prefix of $v^{n}$. Thus $v^{n} \notin \operatorname{Pref}\left((S(A)) \pi^{-1} \iota\right)$. Since $v \pi \in N(A)$ would imply $\left(v^{n}\right) \pi \in S(A)$ and so $v^{n} \in(S(A)) \pi^{-1} \iota$, we conclude that $v \pi \notin N(A)$.

It remains to be considered the case $|X|>1$. Since $u$ and $v$ are both reduced words, there exists some reduced word $w$ such that $u w v w^{-1} u^{-1} \in \bar{X}^{*} \iota$. In fact, we only need to make sure that:

- the first letter of $w$ is not the inverse of the last letter of $u$;

- the last letter of $w$ is not the last letter of $v$;

- the last letter of $w$ is not the inverse of the first letter of $v$.

Since $|\bar{X}| \geq 4$, this can be easily achieved. Suppose that $v \pi \in N(A)$. Then $\left(u w v w^{-1} u^{-1}\right) \pi \in N(A) \subseteq S(A)$ and so

$$
u w v w^{-1} u^{-1}=\left(u w v w^{-1} u^{-1}\right) \iota \in(S(A)) \pi^{-1} \iota .
$$

This contradicts $u \notin \operatorname{Pref}\left((S(A)) \pi^{-1} \iota\right)$, hence $v \pi \notin N(A)$ as required.

In view of Corollaries 4.8 and 5.5, we may now extend Corollary 4.10 to rational languages, providing in the whole an alternative path to yet another Sénizergues result:

Corollary 5.6 [7]. Let $A \in \operatorname{Rat} F G(X)$. Then the syntactic congruence $\sim_{A}$ is decidable and the syntactic group $F G(X) / \sim_{A}$ is effectively constructible. 


\section{REFERENCES}

[1] J. Berstel, Transductions and Context-free Languages. Teubner (1979).

[2] J.C. Birget, S. Margolis, J. Meakin and P. Weil, PSPACE-completeness of certain algorithmic problems on the subgroups of the free groups, in Proc. ICALP 94. Lect. Notes Comput. Sci. (1994) 274-285.

[3] M. Hall Jr., The Theory of Groups. AMS Chelsea Publishing (1959).

[4] R.C. Lyndon and P.E. Schupp, Combinatorial Group Theory. Springer-Verlag (1977).

[5] J. Sakarovitch, Syntaxe des langages de Chomsky, essai sur le déterminisme. Ph.D. thesis, Université Paris VII (1979).

[6] J. Sakarovitch, A problem on rational subsets of the free group. Amer. Math. Monthly 91 (1984) 499-501.

[7] G. Sénizergues, On the rational subsets of the free group. Acta Informatica 33 (1996) 281296.

[8] P.V. Silva, On free inverse monoid languages. RAIRO: Theoret. Informatics Appl. 30 (1996) 349-378.

[9] P.V. Silva, Recognizable subsets of a group: finite extensions and the abelian case. Bulletin of the EATCS $\mathbf{7 7}$ (2002) 195-215.

Communicated by A. Restivo.

Received November 26, 2001. Accepted December 11, 2003.

To access this journal online: www.edpsciences.org 